\section{Subperiosteal hematoma of the orbit associated with subfrontal hematoma presenting as proptosis}

Sir,

Blunt trauma to the head may result in extradural hematomas, which are common over the temporoparietal region and associated with injury to the meningeal arteries. Subperiosteal hematomas of the orbit are rare presentations after head injury, with only a few cases reported in the literature. ${ }^{[1,2]}$ We report a rare case where extradural orbital hematoma presented as proptosis following head injury in a conscious young boy. A 14-year-old boy from rural central India presented with history of fall from the bicycle one day ago. He sustained injury in the form of a scalp contusion over the left frontal region. There was no history of loss of consciousness or any bleed from the oral, nasal or auricular orifices. He had no signs of raised intracranial tension or any neurodeficits. The patient was admitted for observation. Next day he developed proptosis of the left eye, which gradually progressed over $24 \mathrm{~h}$. He also developed throbbing pain over the left orbit and complained of diminution of vision by Day 2. On examination he was fully conscious, with a normal Glasgow coma scale. Bilateral pupils were equal and normally reacting to light. He had restriction of movements of the left eye, with marked limitation of elevation and slight limitation of depression, abduction and adduction. Fundus revealed a hyperemic disc, macular edema, absence of spontaneous venous pulsations and slightly dilated and tortuous retinal veins. He had no cranial nerve palsy or long tract signs. Proptosis of the left eye was observed. X-ray skull revealed no bony abnormality. Computed tomography (CT) of the head demonstrated a large frontal and subfrontal extradural hematoma, which was pushing the left eyeball anteriorly [Figures 1, 2]. No orbital roof fracture was demonstrated. He underwent left frontal craniotomy, superolateral orbitotomy and both hematomas were evacuated. Proptosis resolved immediately after evacuation. Visual acuity returned to normal on the second postoperative day. Postoperative course was uneventful. After four months of follow-up, patient was asymptomatic and attending school. The present case reported is rare, as only a few cases of retroorbital subperiosteal hematomas have been reported previously. All the previous case reports, including the present one have been in young males. This is likely due to higher incidence of trauma in them and also because orbital periosteum is easily detachable. ${ }^{[1,2]}$ Apart from trauma other rare causes of retro-orbital subperiosteal

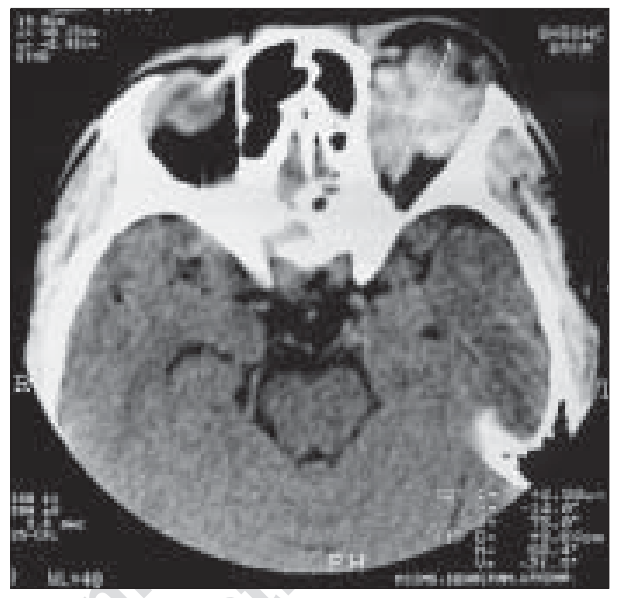

Figure 1: CT scan axial view, showing left retro-orbital hyperdense collection puhing the eyeball anteriorly

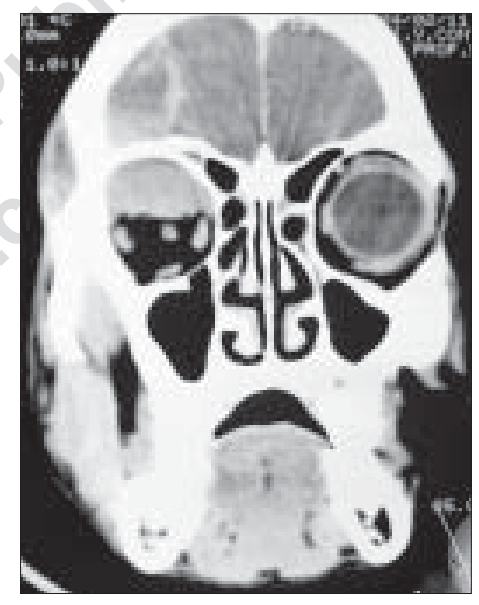

Figure 2: CT scan coronal view, showing left frontal extrduarl hematoma, with subperiosteal collection in left orbit

hematoma include giant cephalhematoma, ${ }^{[3]}$ sickle cell disease ${ }^{[4]}$ and chronic sinusitis. ${ }^{[5]}$ As the proptotic eye is pushed anteriorly against the canthis, pressure within the eye increases until there is inadequate perfusion of the eye due to the pressure effects. This can lead to diminution in vision as in our case. Preservation of consciousness is also rare in previous reported cases. In a series of 10 cases of extradural hematomas with proptosis reported previously, ${ }^{[1]}$ eight had impaired consciousness and associated frontal hematoma. The present case did not have any orbital roof fracture. In a previously reported case series retro-orbital hemorrhage was reported in only two cases and one of them had fracture of the orbital roof. ${ }^{[1]}$ It is important to recognize and treat this condition early, as extradural hematomas have a high mortality. ${ }^{[1,2]}$ Computed tomography scan of the orbit is the most common method of diagnosing a retrobulbar hematoma. Bedside CT scan, in a busy emergency setting ultrasonography may aid in the rapid diagnosis of a retrobulbar hematoma. ${ }^{[5]}$ This case also signifies the importance of prompt surgical intervention, as the patient had a complete recovery. 
Amit Agrawal, Sankalp Dwivedi, Rajnish Joshi*, Dilip Gupta, Mohammed Yunus

Departments of Surgery and *Medicine, Mahatma Gandhi Institute of Medical Sciences, Sevagram Wardha - 442 102, India. E-mail: dramit_in@yahoo.com

\section{References}

1. U mansky F, Pomenanz S. E pidural haematoma and unilateral exophthalmos: A review. Acta N eurochir (Wein) 1989;99:145-7.

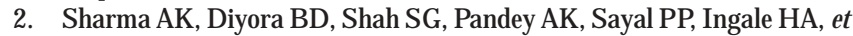
al. Orbital subperiosteal hematoma associated with subfrontal extradural hematoma. J Trauma 2007;62:523-5.

3. Mallouh AA, Young M, Hamdan J, Salamah MM. Proptosis, skull infarction and retro-orbital and epidural hematomas in a child with sickle cell disease. Clin Pediatr (P hila) 1987;26:536-8.

4. I chino $Y, N$ agata $M$, I shikawa $T$. Subperiosteal orbital hemorrhage associated with chronic sinusitis: A case report and review of the literature. Auris Nasus L arynx 1985;12:27-30.

5. Estevez A, Deutch J, Sturmann K, Ziefer B, Ishikawa H, H su C. U Itrasonography evaluation of retrobulbar hematoma in bovine orbits. Acad E merg Med 2000;7:1169-70.

6. Aguas J, Conde C, Fructuoso GG, Mondelo FJ, Ferrer E. Giant cephalhematoma in a 15-year-old boy: U nilateral amaurosis as the main complication. Surg N eurol 1995;43:363-6.

Accepted on 11-04-2007 